(CC BY 4.0) | ISSN 2525-3409 | DOI: http://dx.doi.org/10.33448/rsd-v9i6.3448

\title{
Ensino por Competências na Educação Básica: proposta de um instrumento de avaliação de conteúdo
}

\section{Competency Teaching in Basic Education: proposal for a content assessment tool} Enseñanza de competencias en educación básica: propuesta de herramienta de evaluación de contenido

Recebido: 02/04/2020 | Revisado: 07/04/2020 | Aceito: 15/04/2020 | Publicado: 17/04/2020

\section{Letícia Aparecida Alves de Lima}

ORCID: https://orcid.org/0000-0002-8439-3029 Centro Universitário Internacional (UNINTER), Brasil

E-mail: lealveslima26@gmail.com Marcus Vinicius Gonçalves da Silva ORCID: https://orcid.org/0000-0003-2145-0871 Pontifícia Universidade Católica do Paraná (PUCPR), Brasil E-mail: marvin.gsilva@gmail.com

\section{Resumo}

Competência é definida na Base Nacional Comum Curricular (BNCC) como a mobilização de conhecimentos, habilidades, atitudes e valores para resolver demandas complexas da vida cotidiana, porém não aponta como avaliar as competências na educação básica. Este trabalho, por meio de um estudo de caso e enfoque qualitativo, objetiva propor um instrumento de avaliação de conteúdo, para os alunos do ensino fundamental de um colégio que pertence à rede pública federal de ensino, de forma multidisciplinar. Os resultados demonstram que as competências podem ser mensuradas por meio de um instrumento simples, objetivo e replicável.

Palavras-chave: Competências; BNCC; Educação Básica; Avaliação.

\footnotetext{
Abstract

Competence is defined in the Common National Curricular Base (BNCC) as the mobilization of knowledge, skills, attitudes and values to solve complex demands of everyday life however, it does not point out how to assess competences in basic education. In this job, through a case study and and qualitative approach, aims to propose a content evaluation
} 
instrument, for elementary school students at a college that belongs to the federal public school system, in a multidisciplinary way. The results demonstrate that competences can be measured using a simple, objective and replicable instrument.

Keywords: Competence; BNCC; Basic Education; Evaluation.

\section{Resumen}

La competencia se define en la Base Curricular Común Nacional (BNCC) como la movilización de conocimientos, habilidades, actitudes y valores para resolver demandas complejas de la vida cotidiana, sin embargo, no señala cómo evaluar las competencias en educación básica. En este trabajo, a través de un estudio de caso y enfoque cualitativo, pretende proponer un instrumento de evaluación de contenido, para estudiantes de primaria en una universidad que pertenece al sistema de escuelas públicas federales. Los resultados demuestran que las competencias se pueden medir utilizando un instrumento simple, objetivo y replicable.

Palabras clave: Habilidades; BNCC; Educación básica; Evaluación.

\section{Introdução}

Ao se falar em modelo de ensino por competências busca-se integrar os saberes em todas as suas dimensões. Desse modo, a competência consiste na intervenção eficaz nos diferentes âmbitos da vida, mediante ações nas quais se mobilizam, ao mesmo tempo e de maneira inter-relacionada, componentes conceituais (saber), procedimentais (saber fazer) e atitudinais (ser) (Zabala, 1998).

A discussão acerca da avaliação da aprendizagem implica em falar de mudanças e transformações de métodos tradicionais, por vezes, considerada seletiva, excludente e realizada de forma soberana sobre os educandos (Do Prado, et al., 2012).

Cabe ter em vista que o conteúdo escolar não envolve apenas os conceitos, mas procedimentos e atitudes que devem estar inseridos no processo de ensino e aprendizagem de forma inter-relacionados (Coll, et al., 1992 apud Volpato, et al., 2017).

Nesse contexto, urge a seguinte questão de pesquisa: Como avaliar as competências na Educação Básica?

Para responder à essa questão, por meio de um estudo de caso, o trabalho objetiva propor um instrumento de avaliação de conteúdo para os alunos do $6^{\circ}$ ano, de forma 
multidisciplinar, e os resultados demonstram que as competências podem ser mensuradas por meio de um instrumento simples, objetivo e replicável.

\section{Fundamentação Teórica}

Na Base Nacional Comum Curricular (BNCC), competência é definida como a "mobilização de conhecimentos (conceitos e procedimentos), habilidades (práticas, cognitivas e socioemocionais), atitudes e valores para resolver demandas complexas da vida cotidiana, do pleno exercício da cidadania e do mundo do trabalho" (Brasil, 2017).

De modo geral, as competências na Educação Básica se inter-relacionam e desdobramse no tratamento didático proposto para as três etapas da Educação Básica (Educação Infantil, Ensino Fundamental e Ensino Médio), articulando-se na construção de conhecimentos, no desenvolvimento de habilidades e na formação de atitudes e valores, nos termos da Lei de Diretrizes e Bases (Brasil, 1996).

Para Machado (2009 apud Freitas \& Aquino, 2020, p. 10), ao "desenvolver competências, a escola deve considerar que essas não são simples resultados da adição dos saberes, tampouco se limitariam ao domínio de saberes". Para tanto, torna-se necessária uma maior integração de diferentes recursos, a fim de buscar superar o conteudismo.

Perrenoud (1999) revela que as competências mobilizam conhecimentos, em grande parte de ordem disciplinar, até que a organização diferencie as disciplinas, e que cada uma assuma um nível ou um componente da realidade.

Nesse contexto, as competências se referem ao desenvolvimento dos estudantes ao longo de sua escolaridade, numa concepção de formação integral do ser humano, pois a BNCC aponta que os alunos devam articular conhecimentos, habilidades, atitudes e valores de modo a participar ativamente da vida em sociedade, seja no mundo do trabalho ou como cidadãos.

\section{Metodologia}

A metodologia adotada no trabalho tem abordagem exploratória e descritiva, de natureza qualitativa, e de um estudo de caso realizado junto aos alunos do $6^{\circ}$ ano da educação básica de uma colégio pertencente a uma rede pública federal de ensino.

Pereira et al. (2018) descrevem que nos estudos exploratórios uma forma de investigação muito utilizada é o estudo de caso, ou seja, um método de estudo de fenômenos 
individuais ou processos sociais. Neste trabalho, o processo social analisado é o de avaliação das competências.

\subsection{Categorias de análise}

Os conteúdos de aprendizagem não se reduzem unicamente às contribuições das disciplinas ou matérias tradicionais. Portanto, também serão conteúdos de aprendizagem todos aqueles que possibilitem o desenvolvimento das capacidades motoras, afetivas, de relação interpessoal e de inserção social.

Quando a função social que se atribui ao ensino é a formação do ser, e a concepção sobre os processos de ensino/aprendizagem é construtivista, e de atenção a diversidade, os resultados do modelo teórico tendem a não ser tão uniformes como no modelo tradicional.

Em consonância com a definição de competência elencada na BNCC, neste trabalho optou-se pela abordagem dos conteúdos propostos por Zabala (1998), o qual os divide em três categorias: conceituais, procedimentais e atitudinais, descritas nos tópicos seguintes.

\subsubsection{Conteúdos conceituais}

Para Zabala (1998, p. 43), do ponto de vista educacional, o conhecimento do aluno não deve ser mensurado por sua capacidade de repetir uma definição, mas quando sabe utilizá-la para interpretar e compreender um fenômeno ou situação e, quando é capaz de situar os fatos, objetos ou situações concretas naquele conceito que os inclui. Assim, o conteúdo conceitual refere-se ao aprendizado que permite uma compreensão que vai além da mera reprodução de enunciados mais ou menos literais.

\subsubsection{Conteúdos procedimentais}

Zabala (1998, p. 44) descreve que os conteúdos procedimentais são um conjunto de ações ordenadas dirigidas para a realização de um objetivo, e que podem ser situadas em três eixos: i) motor/cognitivo; ii) poucas ações/muitas ações; e, iii) continuum algorítmico/heurístico. Saltar ou recortar são exemplos do eixo motor; enquanto inferir, ler ou traduzir, mais próximos do cognitivo. Realizar um cálculo, situa-se no eixo poucas ações; enquanto ler e desenhar se encontrariam mais próximos do eixo muitas ações. O continuum algorítmico/heurístico refere-se ao grau de determinação da ordem das sequencias.

Mais próximo do extremo algorítmico, estariam os conteúdos cuja ordem das ações se repetem de maneira constante, enquanto no eixo heurístico estariam os conteúdos cujas ações 
a serem realizadas e a maneira de organizá-las dependem das características da situação em que se deve aplicá-los (Zabala, 1998). Nesse contexto, os procedimentos a serem realizados não devem exigir as mesmas ações, de forma que o ponto de partida é a estratégia procedimental.

\subsubsection{Conteúdos atitudinais}

Zabala (1998) pontua que os conteúdos atitudinais englobam uma série de conteúdos agrupados em valores, atitudes e normas.

Os valores são princípios ou ideias éticas que permitem que as pessoas emitam um juízo de valor sobre as condutas e seu sentido. As atitudes referem-se às tendências ou predisposições relativamente estáveis das pessoas para atuar de certa maneira. As normas correspondem aos padrões ou regras de comportamento que devemos seguir em determinadas situações que obrigam a todos os membros de um grupo social (Zabala, 1998).

No meio escolar, os conteúdos atitudinais são trabalhados, ainda que, espontaneamente, nas atividades individuais ou em grupos, sendo que nestes últimos, há uma melhor observação, pois se propõe a convivência em constante integração, buscando fortalecer o respeito mútuo na pluralidade e divergência de opiniões e os valores socialmente estabelecidos.

\subsection{Coleta dos Dados}

A coleta dos dados foi realizada por meio de um instrumento elaborado neste estudo, denominado - Ficha de Avaliação de Conteúdos (Figura 1). Os alunos foram divididos em 10 (dez) grupos.

A operacionalização do instrumento proposto permitiu para avaliar as competências na atividade desenvolvida em sala de aula sobre o tema Meio Ambiente, de modo interdisciplinar. Na disciplina de Língua Portuguesa foi trabalhado o gênero textual - notícia e reportagem - e, na disciplina de Ciências, as especificidades do tema.

Ao todo, foram propostos 23 critérios de avaliação dos conteúdos, totalizando 69 escores (27-conceituais, 21-procedimentais e 21-atitudinais), sendo que cada escore equivale a 0,1449 ponto para a composição da nota, isto é, 69 escores corresponderam à nota 10,0, sendo os demais escores calculado por meio do método resolutivo da regra de três simples, observando-se as regras de arredondamento. 
Research, Society and Development, v. 9, n. 6, e84963448, 2020

(CC BY 4.0) | ISSN 2525-3409 | DOI: http://dx.doi.org/10.33448/rsd-v9i6.3448

Na legenda da Figura 1, são descritos os critérios de avaliação conforme a nota atribuída numa escala de 0 a 4.

Figura 1. Ficha de Avaliação de Conteúdos.

\begin{tabular}{|c|c|c|c|c|c|c|c|}
\hline \multicolumn{8}{|c|}{$\begin{array}{c}\text { Sequência Didática Interdisciplinar }-6^{\circ} \text { ano - } 2019 \\
\text { Língua Portuguesa - Ciências }\end{array}$} \\
\hline \multicolumn{8}{|c|}{$\begin{array}{l}\text { Eixo Transversal: Meio Ambiente } \\
\text { Situação-Problema: Meio ambiente é um conjunto de unidades ecológicas que funcionam como um sistema natural, e incluem } \\
\text { toda a vegetação, animais, microrganismos, solo, rochas, atmosfera e fenômenos naturais que podem ocorrer em seus limites. Os } \\
\text { desafios expressos por cientistas ambientais incluem: } \\
\text { - Redução e limpeza da poluição, com metas futuras de poluição zero; } \\
\text { - Reduzir o consumo combustiveis não-renováveis; } \\
\text { - Desenvolvimento de fontes de energia alternativas, verdes, com pouco carbono ou de } \\
\text { energia renovável; } \\
\text { - Conservação e uso sustentável dos escassos recursos naturais como água, terra e ar; } \\
\text { - Proteção de ecossistemas representativos ou únicos; } \\
\text { - Oreservação de espécie em perigo ou ameaçadas de extinção; } \\
\text { vidas na Terra dependem. }\end{array}$} \\
\hline Conteúdos & Critérios de Avaliação & N. A & 3 & 2 & 1 & 0 & TOTAL \\
\hline \multirow{9}{*}{$\begin{array}{l}\text { Conceituais } \\
\text { GBO } 27 \text { escores }\end{array}$} & Originalidade & & & & & & \\
\hline & Utilizou o Gênero Reportagem/Notícia & & & & & & \\
\hline & Escolha do Tema (Meio Ambiente) & & & & & & \\
\hline & Abordagem do tema & & & & & & \\
\hline & Tempo minimo de 5 minutos & & & & & & \\
\hline & Utilizou a norma culta da Língua Portuguesa & & & & & & \\
\hline & Utilizou a linguagem científica das Ciências & & & & & & \\
\hline & Utilizou os conceitos empregados nas aulas de Ciências/Port. & & & & & & \\
\hline & Referências & & & & & & \\
\hline \multirow{7}{*}{$\begin{array}{l}\text { Procedimentais } \\
\text { GBO } 21 \text { escores }\end{array}$} & Apresentação Escrita/Roteiro & & & & & & \\
\hline & Edição do vídeo & & & & & & \\
\hline & Criatividade & & & & & & \\
\hline & Caracterização & & & & & & \\
\hline & Funções definidas dentro do grupo & & & & & & \\
\hline & Organização do Grupo & & & & & & \\
\hline & Foram apresentadas soluções para os problemas & & & & & & \\
\hline \multirow{7}{*}{$\begin{array}{l}\text { Atitudinais } \\
\text { GBO } 21 \text { escores }\end{array}$} & Interação & & & & & & \\
\hline & Trabalho em Grupo & & & & & & \\
\hline & Pontualidade & & & & & & \\
\hline & Responsabilidade & & & & & & \\
\hline & Tolerância & & & & & & \\
\hline & Autonomia & & & & & & \\
\hline & A reportagem/notícia do vídeo despertou conscientização & & & & & & \\
\hline
\end{tabular}

Legenda:

0 - Não realizou a tarefa.

1- Realizou a tarefa sem atender aos critérios estabelecidos.

2- Realizou a tarefa atendendo parcialmente aos critérios estabelecidos.

3- Realizou a tarefa atendendo todos os critérios estabelecidos.

4- Não avaliado.

Fonte: Elaboração própria.

Certamente, a distribuição da importância relativa dos distintos conteúdos não é a mesma em cada um dos diferentes períodos. O mais provável é que nos cursos iniciais exista 
urna distribuição mais equilibrada dos diversos conteúdos, ou que se dê prioridade aos procedimentais e atitudinais acima dos conceituais, e que à medida que vai se avançando nos níveis de escolarização se incremente o peso dos conteúdos conceituais em detrimento dos procedimentais e atitudinais.

\section{Resultados e Discussões}

Conforme se observa no Gráfico 1, as competências inerentes aos 10 (dez) grupos de alunos na atividade relacionada ao tema Meio Ambiente foram avaliadas em relação aos conteúdos conceituais, procedimentais e atitudinais, propostos por Zabala (1998). A fim de facilitar a compreensão, o gráfico apresenta o número de escores obtidos e a conversão dos escores em notas.

Gráfico 1. Escores e Notas obtidas pelos grupos nos conteúdos

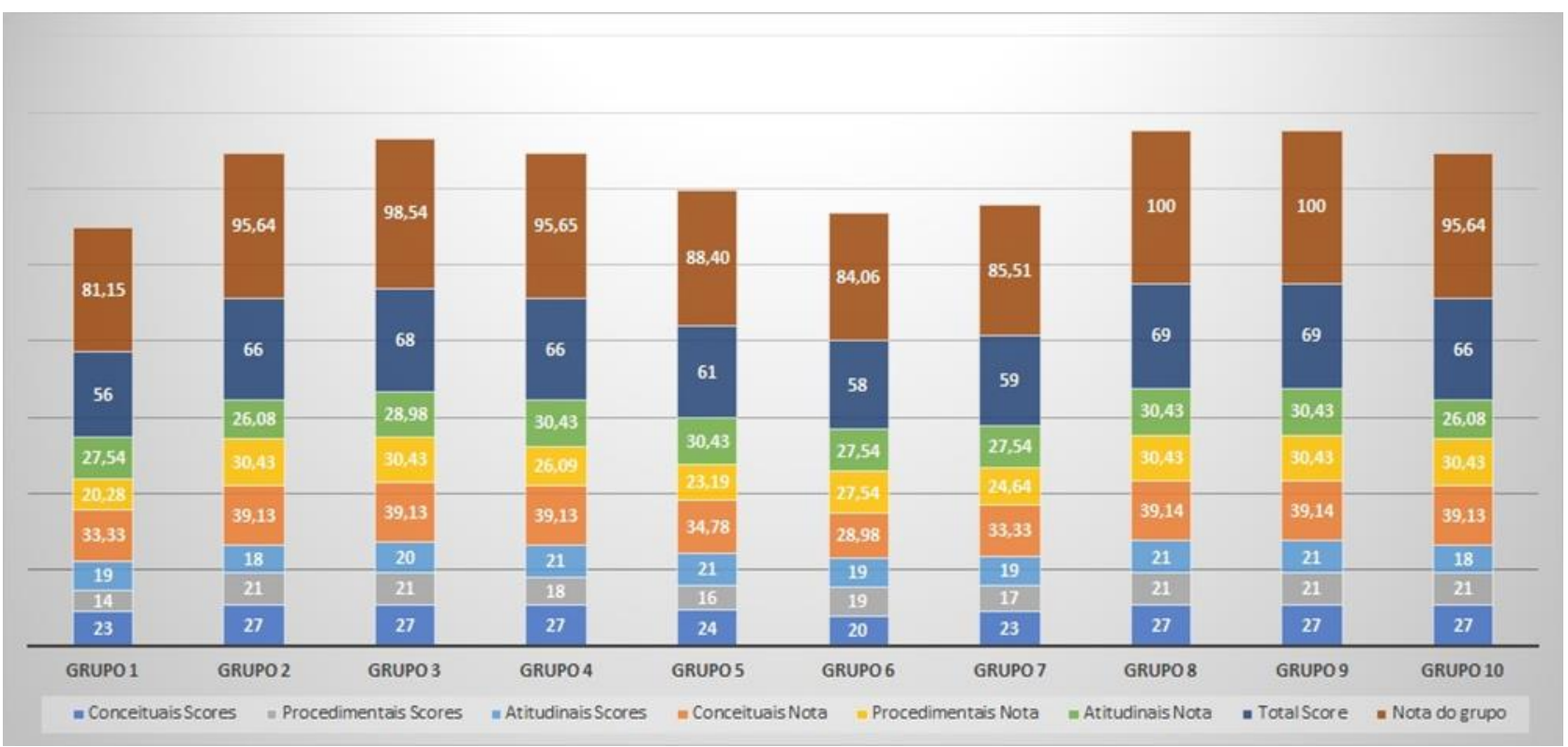

Fonte: elaboração própria.

A tendência é de situar os diferentes conteúdos sob a perspectiva disciplinar ou a área de ensino: português, língua, matemática, música, geografia etc., produzindo, ao mesmo tempo, didáticas específicas de cada disciplina. 
Research, Society and Development, v. 9, n. 6, e84963448, 2020

(CC BY 4.0) | ISSN 2525-3409 | DOI: http://dx.doi.org/10.33448/rsd-v9i6.3448

O parâmetro utilizado na análise foi a média geral obtida pelos grupos em relação aos conteúdos (Gráfico 2).

Gráfico 2. Média geral obtida pelos grupos.

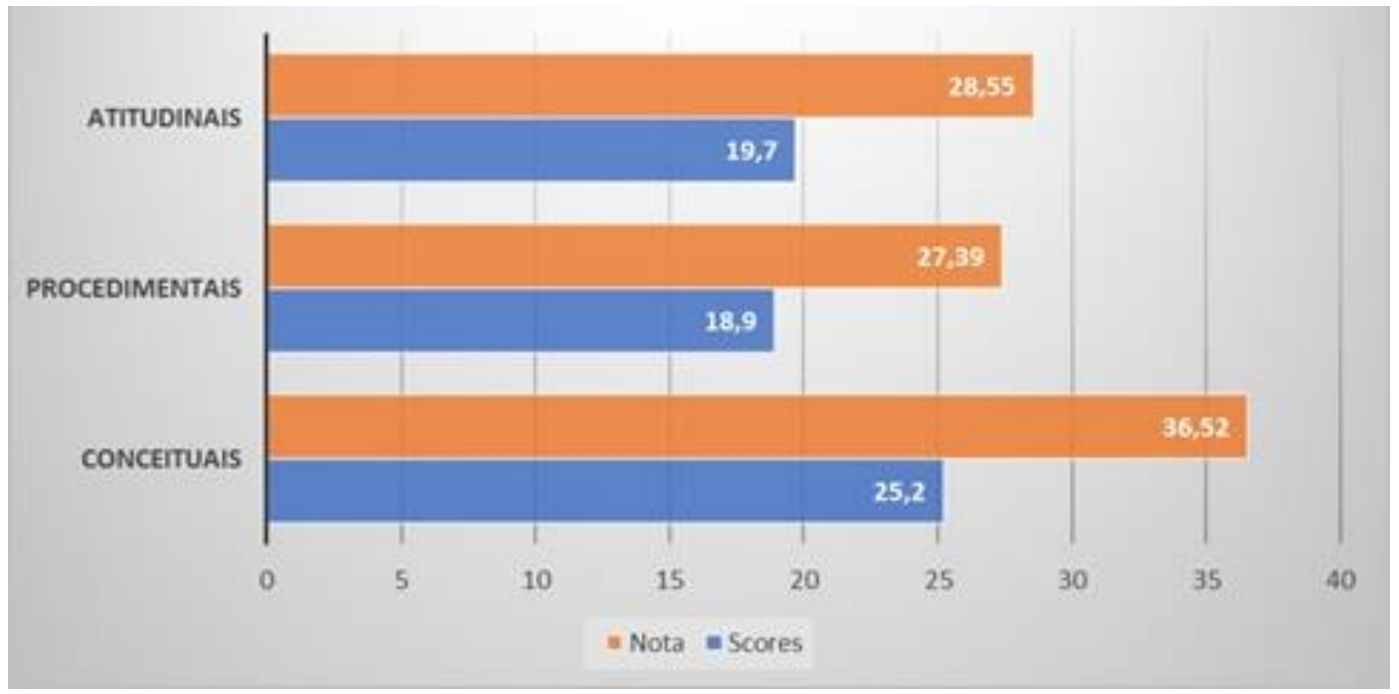

Fonte: elaboração própria.

Demonstrado nos gráficos 1 e 2, os escores obtidos nos conteúdos conceituais compuseram a maior parte da nota dos grupos, com uma média de 36,52, sendo que os grupos 1, 5, 6 e 7 ficaram abaixo da média, ou seja, 40\%. Nota-se, que em relação aos conteúdos procedimentais, os grupos 1, 5, 6 e 7, também ficaram com a nota abaixo da média dos outros grupos $(27,39)$, o que denota, em tese, certa relação entre os conteúdos conceituais e procedimentais. Por fim, na avaliação dos conteúdos atitudinais, os grupos 1, 2, 6, 7 e 10 obtiveram nota um pouco inferior da média $(28,55)$.

Esses dados numéricos permitem avaliar o panorama da turma no que denominamos CHA da competência do aprendizado, um acrônimo para conhecimentos, habilidades e atitudes, posteriormente analisados de forma qualitativa.

Seguramente, a classificação da importância relativa aos conteúdos não é a mesma em cada uma das diferentes séries do ensino. O mais aceitável é que nos cursos iniciais exista uma distribuição mais equilibrada dos diversos conteúdos, ou que se priorize os procedimentais e atitudinais em relação aos conceituais, e à medida que se avança nos níveis de escolarização, se aumente o peso dos conteúdos conceituais. 


\section{Considerações Finais}

Este trabalho permitiu demonstrar que as competências dos alunos das séries iniciais da educação básica podem ser mensuradas por meio de um instrumento de avaliação de conteúdo, de modo simples, objetivo e replicável.

Para tanto, a escola deve incentivar a ensino por competências, imprescindível para o desenvolvimento pessoal, interpessoal e social, congruente com a proposta de Zabala e Arnau (2010, p. 22) no sentido de "buscar superar a função propedêutica e seletiva do ensino tradicional".

A avaliação dos conteúdos não é um processo em si mesmo, tampouco um fim, mas um método que deve ser aplicado de forma contínua, cujo objetivo é propiciar a compreensão dos limites e avanços na construção do aprendizado dos alunos, contribuindo com o desenvolvimento de suas competências.

Aponta-se como limitação do estudo, a aplicação do instrumento apenas junto a alunos do $6^{\circ}$ ano do ensino fundamental da educação básica, porém não o invalida, pois o objetivo proposto foi alcançado - propor um instrumento de avaliação de conteúdo.

Desse modo, como recomendações para estudos futuros, sugere-se a aplicabilidade do instrumento em outros estabelecimentos de ensino da rede estadual e municipal, e em outras séries da educação básica, a fim de que os resultados deste trabalho possam ser corroborados ou sejam identificados novos achados.

\section{Referências}

Brasil. (2017). Resolução CNE/CP nº 2, de 22 dez. 2017. Institui e orienta a implantação da Base Nacional Comum Curricular, a ser respeitada obrigatoriamente ao longo das etapas $e$ respectivas modalidades no âmbito da Educação Básica. Disponível em:

<http://portal.mec.gov.br/index.php?option=com_docman\&view=download\&alias=79631rcp002-17-pdf\&category_slug=dezembro-2017-pdf\&Itemid=30192>. Acesso em: 25 mar. 2020.

Brasil. (1996). Lei no 9.394, de 20 dez. 1996. Estabelece as diretrizes e bases da educação nacional. Diário Oficial da União, Brasília, 23 de dezembro de 1996. Disponível em: <http://www.planalto.gov.br/ccivil_03/leis/L9394.htm>. Acesso em: 26 mar. 2020. 
Do Prado, R. A., Do Prado, M. L. \& Reibnitz, K. S. (2012). Desvelando o significado da avaliação no ensino por competência para enfermeiros educadores. Rev. Eletrônica de Enfermagem, v. 14, n. 1, p. 112-21, 2012. Disponível em: https://www.fen.ufg.br/revista/v14/n1/pdf/v14n1a13.pdf Acesso em: 13 mar. 2020.

Freitas, S., \& Aquino, F. (2020). The pedagogy of competences in the Institutional Pedagogical Political Project of the Federal Institute of Education, Science and Technology of Ceará: a brief analysis. Research, Society and Development, 9(3), e100932607. doi: http://dx.doi.org/10.33448/rsd-v9i3.2607

Pereira, A. S., et al. (2018). Metodologia da pesquisa científica. [e-book]. Santa Maria. Ed. UAB/NTE/UFSM. Disponível em:

https://repositorio.ufsm.br/bitstream/handle/1/15824/Lic_Computacao_MetodologiaPesquisa-Cientifica.pdf?sequence=1. Acesso em: 13 mar. 2020.

Perrenoud, P. (1999). Construir as competências desde a escola. Porto Alegre, Artmed, 96 p.

Volpato, V. C.; Aguiar, J. A. \& Dos Reis, J. M. C. (2017). A construção de conhecimentos conceituais, procedimentais e atitudinais: contribuições de uma oficina temática sobre investigação criminal. Actio: Docência em Ciências, 2(3). doi: 10.3895/actio.v2n3.6863.

Zabala, A \& Arnau, L. (2010). Como aprender e ensinar competências. Tradução de Carlos Henrique Lucas Lima. Porto Alegre: Artmed, 197 p., 2010.

Zabala, A. (1998). A prática educativa: como ensinar. Porto Alegre: Artmed, 1998, 224 p.

\section{Porcentagem de contribuição de cada autor no manuscrito}

Letícia Aparecida Alves de Lima - 60\%

Marcus Vinicius Gonçalves da Silva - 40\% 